https://helda.helsinki.fi

\title{
Characterization of an X-chromosome-linked telomere biology disorder in females with DKC1 mutation
}

Hirvonen, Elina A. M.

2019-01

Hirvonen, E A M , Peuhkuri , S, Norberg , A , Degerman , S , Hannula-Jouppi , K, Välimaa , H , Kilpivaara , O \& Wartiovaara-Kautto , U 2019 , ' Characterization of an X-chromosome-linked telomere biology disorder in females with DKC1 mutation ', Leukemia , vol. 33 , no. 1 , pp. 275-278 . https://doi.org/10.1038/s41375-018-0243-5

http://hdl.handle.net/10138/310701

https://doi.org/10.1038/s41375-018-0243-5

publishedVersion

Downloaded from Helda, University of Helsinki institutional repository.

This is an electronic reprint of the original article.

This reprint may differ from the original in pagination and typographic detail.

Please cite the original version. 
Leukemia (2019) 33:275-278

https://doi.org/10.1038/s41375-018-0243-5

Cytogenetics and molecular genetics

\title{
Characterization of an X-chromosome-linked telomere biology disorder in females with DKC1 mutation
}

\author{
Elina A. M. Hirvonen ${ }^{1}$ - Saara Peuhkuri ${ }^{1} \cdot$ Anna Norberg $^{2}$ - Sofie Degerman $\mathbb{1}^{2} \cdot$ Katariina Hannula-Jouppi $^{3,4}$. \\ Hannamari Välimaa ${ }^{5}$ - Outi Kilpivaara ${ }^{1}$ • Ulla Wartiovaara-Kautto ${ }^{6}$
}

Received: 18 June 2018 / Revised: 13 July 2018 / Accepted: 19 July 2018 / Published online: 5 September 2018

(c) Springer Nature Limited 2018

Dyskeratosis congenita (DC) caused by mutations in genes implicated in telomere biology is an inherited syndrome affecting multiple tissues. Clinical severity and the spectrum of symptoms are variable but classically patients present with significant hemato-immunological, odontological, mucosal, and dermatological disturbances [1]. This is due to defective telomere maintenance in rapidly renewing tissues, causing shortened telomeres and DC-like manifestations in the respective organ. Other common clinical features are hepatic, pulmonal, ophthalmologic, and neuropsychiatric health problems. DC also predisposes patients to cancer, especially hematological malignancies and squamous cell carcinomas of the head and neck [2]. Remarkably, the risk of myelodysplastic syndrome (MDS) is over 500-fold in comparison to healthy individuals [2]. Hence, early recognition of all patients with DC or other telomere biology disorder (TBD) is crucial.

One of the genes linked to DC is $D K C 1$, located on chromosome $\mathrm{X}$ and encoding a highly conserved protein dyskerin. Dyskerin is an essential nucleolar protein, which

These authors contributed equally: Outi Kilpivaara, Ulla WartiovaaraKautto.

Electronic supplementary material The online version of this article (https://doi.org/10.1038/s41375-018-0243-5) contains supplementary material, which is available to authorized users.

Outi Kilpivaara

outi.kilpivaara@helsinki.fi

$\bowtie$ Ulla Wartiovaara-Kautto

ulla.wartiovaara-kautto@hus.fi

1 Genome-Scale Biology/Research Programs Unit, and Department of Medical and Clinical Genetics/Medicum, University of Helsinki, Helsinki, Finland

2 Department of Medical Biosciences, Medical and Clinical Genetics, Umeå University, Umeå, Sweden

3 Department of Dermatology and Allergology, University of maintains stability of the human telomerase RNA (TERC) by interacting with H/ACA consensus sequence in TERC. Pathogenic mutations in $D K C 1$ result in a dysfunctional protein, leading to reduced levels of TERC, decreased telomerase activity, and premature telomere shortening in males [3]. Dyskerin has a role also in controlling ribosome biogenesis [3]. X-chromosome inactivation (XCI) silences transcription from one of the two $\mathrm{X}$ chromosomes in female cells to balance expression dosage between males and females. The inactivation process is sometimes incomplete in human tissues, thus up to one-third of X-chromosomal genes may be expressed from both the active and silenced $\mathrm{X}$ chromosomes [3]. There are few reports on females with DKC1 mutation [4-6]. Here, we report results of detailed molecular and clinical investigation of three symptomatic sisters aiming at enlightening X-linked TBD in females.

We recently identified two brothers with a novel $D K C 1$ mutation c.1218_1219insCAG, p.(Asp406_Ser407insGln) resulting in short telomeres and manifestation of DC [6]. Three female siblings (mother and two aunts of the brothers) were studied at the Department of Hematology in Helsinki University Hospital Comprehensive Cancer Center in 2016-2017 amid hematologic consultation of the brothers. Clinical examination and sampling for extended routine analyses, as well as research laboratory analyses, were performed for all the females. Informed consents were

Helsinki and Helsinki University Central Hospital, Helsinki, Finland

4 Folkhälsan Institute of Genetics, Helsinki, and Molecular Neurology/Research Programs Unit, University of Helsinki, Helsinki, Finland

5 Department of Virology, University of Helsinki and Department of Oral and Maxillofacial Surgery, University of Helsinki and Helsinki University Hospital, Helsinki, Finland

6 Department of Hematology, Helsinki University Hospital Comprehensive Cancer Center and University of Helsinki, Helsinki, Finland 


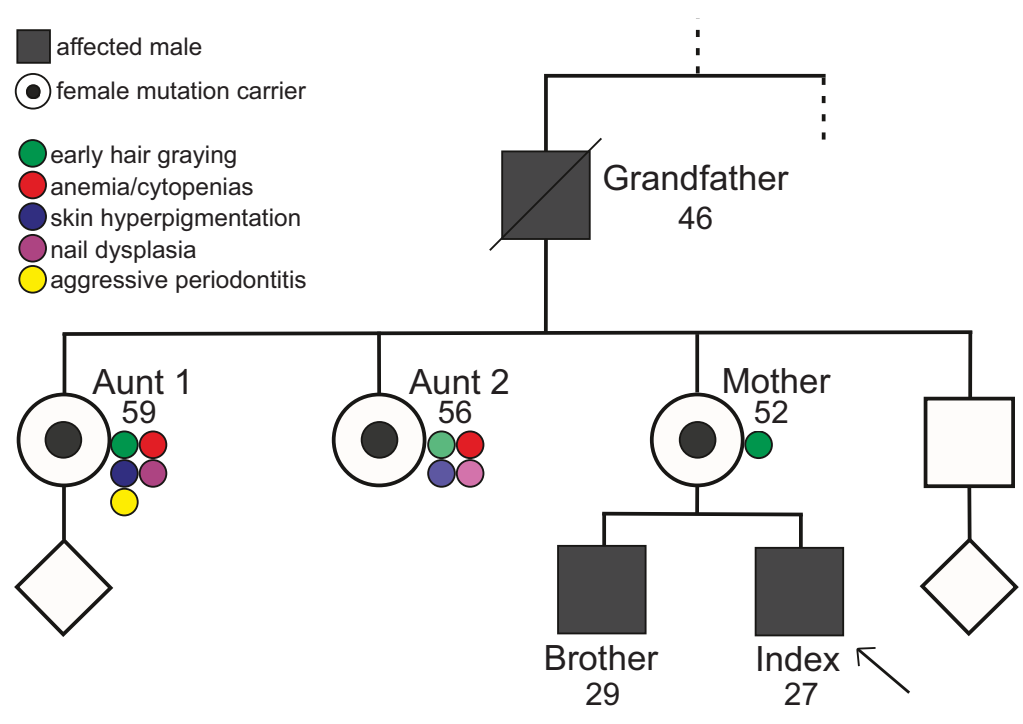

\begin{tabular}{|l|c|c|c|}
\hline $\begin{array}{l}\text { Laboratory findings (reference } \\
\text { values; F=female) }\end{array}$ & Mother & Aunt 1 & Aunt 2 \\
\hline Hb(F: 134-167 g/L) & 141 & 114 & 109 \\
\hline Eryt(F: 4.25-5.70 E12/L) & 4.19 & 3.44 & 3.46 \\
\hline Reticulocytes(0.7-2.3\%) & 1.2 & 2.7 & 1.2 \\
\hline MCV(82-98 fl) & 97 & 98 & 95 \\
\hline Leuk(3.4-8.2 E9/L) & 9.0 & 6.1 & 4.2 \\
\hline Neut(1.5-6.7 E9/L) & 6.6 & 3.7 & 2.4 \\
\hline Lymph(1.3-3.6 E9/L) & 1.5 & 1.9 & 1.3 \\
\hline B-cells(CD19+) (0.08-0.62 E9/L) & 0.23 & 0.19 & 0.27 \\
\hline T-cells(CD3+) (0.75-2.76 E9/L) & 1.14 & 1.22 & 0.84 \\
\hline T-cells(CD4+) (0.404-1.612 E9/L) & 0.502 & 0.744 & 0.268 \\
\hline T-cells(CD8+) (0.22-1.13 E9/L) & 0.59 & 0.50 & 0.58 \\
\hline NK-cells(0.08-0.72 E9/L) & 0.10 & 0.18 & 0.17 \\
\hline HbF(<1.0\%) & 7.4 & 2.9 & 2.0 \\
\hline
\end{tabular}

Fig. 1 Pedigree, clinical features, and laboratory findings of c.1218_1219insCAG, p.(Asp406_Ser407insGln) mutation carrier females. In the pedigree, circles with dots represent mutation carrier females and dark squares represent males with DC. Index case is marked with an arrow. Ages at time of examination are shown. Grandfather died traumatically at 46 years of age. Colored dots denote

obtained from participating individuals. This study was conducted in accordance with the principles of Helsinki Declaration and was approved by the Ethics Committee of Helsinki University Hospital (\#206/13/03/03/2016). Detailed descriptions of the study subjects and analyses are presented in Fig. 1, Supplementary Table 1, and in the Supplementary information.

All the females were confirmed to be heterozygous $D K C 1$ mutation carriers by Sanger sequencing. In order to study $\mathrm{X}$-inactivation in various tissues, we compared the $D K C 1$ expression levels and distribution of wild type (WT) and mutant alleles in blood, buccal mucosa, tongue, and Epstein-Barr virus (EBV)-transformed lymphocytes using Sanger sequencing and droplet digital PCR (ddPCR). ddPCR is superior to traditional quantitative PCR as it is more sensitive and allows absolute quantitative measurement of RNA expression [7] (Fig. 2). With ddPCR, the mutant allele burden in blood was $0 \%$ in the mother, $45 \%$ in aunt 1 , and $5 \%$ in aunt 2 . Also, buccal mucosa and tongue samples showed varying amounts of mutant $D K C 1$ allele expression in all the females (Fig. 2). Although we observed both mutant and WT alleles to be present, e.g., in blood samples, it is possible, or even likely, that signals come from different cells expressing either WT or mutant $D K C 1$, and not both. The EBV-transformed lymphocytes showed extremely high overall $D K C 1$ expression, $>98 \%$ being WT DKC1 (Supplementary Figure 1). Dyskerin expression has been reported to be similar in EBV-transformed lymphoblastoid cells in mutation carrying females vs. controls [8]. the clinical features; darker tone of a colored dot refers to more severe symptoms. Green: early hair graying; red: anemia/cytopenias; blue: skin hyperpigmentation; purple: nail dysplasia; yellow: aggressive periodontitis. Abnormal laboratory findings are shown in red in the table

Here, we show that the extremely high $D K C l$ expression in transformed cells does not reflect the situation in naïve cells. This is not surprising as such, since dyskerin expression increases with malignant transformation [9]. Consequently, EBV-transformed lymphoblastoid cells should not be used in evaluation of telomere functionality.

The telomere lengths (TL) were measured in whole blood by a quantitative PCR-based method [10]. Two out of the three females (mother and the aunt 1) had shortened telomeres compared to age-matched controls (Supplementary Figure 2). The telomeres in the two brothers were previously shown to be very short $(<5$ th percentile) and are depicted in the same figure for comparison. According to the literature, there is not a strict relationship between TL and severity of symptoms at an individual level [11]. Families have been reported where individuals display variable symptoms and differences in telomere lengths even though they carry the same mutation $[12,13]$. Also, gene/ mutation-specific effect on TL is unclear. The brothers with extremely short TL had clear clinical mucocutaneous and immunological manifestations of DC. However, in the females, the correlation of TL with clinical presentation was less obvious and inquired more detailed investigation. The expression of $\mathrm{DKCl}$ measured by ddPCR in blood correlated better with, e.g., anemia.

In $D K C 1$-linked TBD, skewed $\mathrm{X}$-inactivation has been thought to function especially well in hematopoietic tissues [6] and protect females from hematologic symptoms. In our study, both mutant and WT $D K C l$ are expressed in blood in 


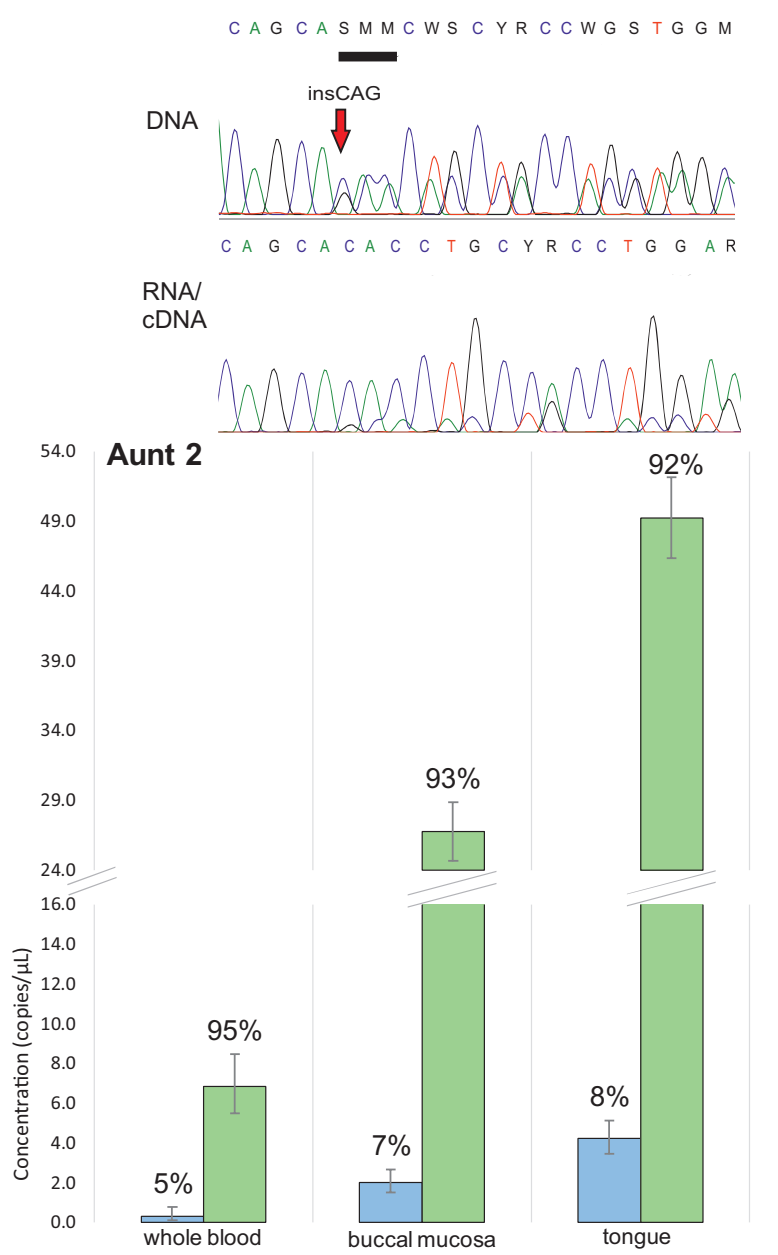

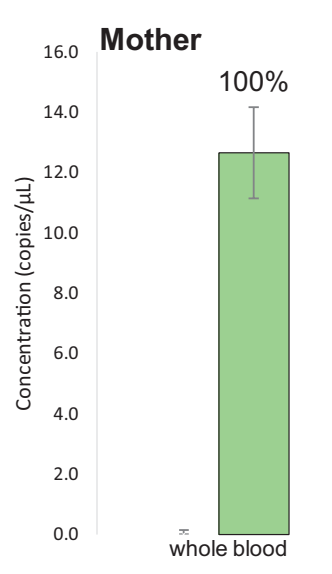

Aunt 1
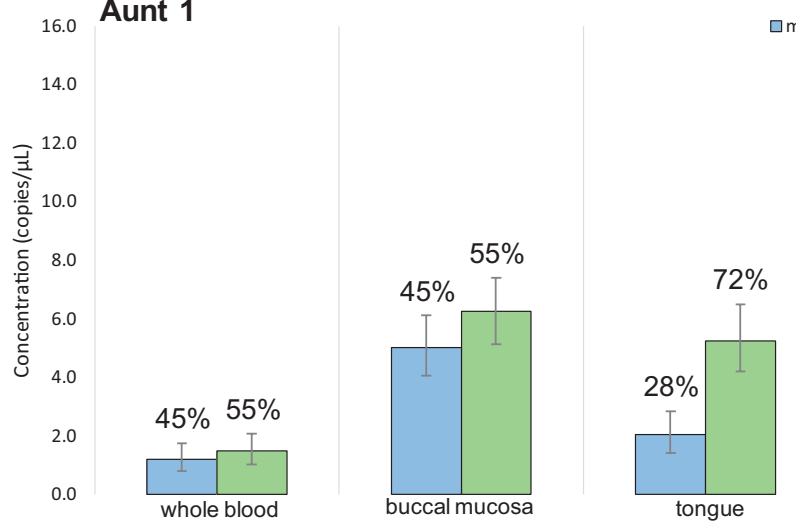

DNA sequence is marked with an arrow. Concentrations (copies/ $\mu \mathrm{l})$ of mut and WT cDNA copies reverse-transcribed from tissue sample RNA from all the females are shown (note different $y$-axis scales). The error bars represent Poisson 95\% confidence intervals

surveillance of genomic evolution in DC patients is still indicated aiming at early detection of MDS.

Regarding other clinical findings in our study patients, at least two of the three females had abnormal early graying of hair and nail atypia (Supplementary Table 1). Also, subtle dermatologic features indicative of DC were present in both aunts (Supplementary Figure 3). However, they lacked the typical reticular pigmentation of the head and neck.

In summary, we demonstrate here that in rare diseases such as TBDs, careful clinical and molecular examination of one family can significantly increase our knowledge on disease features. Despite X-linked inheritance, two out of three females in this study fulfill the diagnostic criteria for DC- or DC-like syndrome [1]. In conjunction with widening the clinical spectrum of $D K C l$-linked TBD in women, we are also able to reveal the molecular roots of patients' symptoms. Whether the unique distribution pattern of mutant and WT $\mathrm{DKCl}$ in distinct tissues arises by cocorresponding well to recent findings [14]. A long-term 
incidence or is a controlled biological phenomenon, remains to be investigated. DC is a multi-organ disease with high incidence of developing malignancies [2]. Since the health problems in female $D K C 1$ mutation carriers may also be significant, the identification, registration, and careful follow-up of these individuals is called for.

Acknowledgements This study was funded by grants from Academy of Finland (\#274474, \#312492, and \#284538), Helsinki University Hospital Comprehensive Cancer Center Research Funding, Cancer Society of Finland, Signe and Ane Gyllenberg Foundation, and Väre Foundation for Pediatric Cancer Research. We are grateful to patients who participated and thus made this study possible. We thank Lotta Honkala, Pihla Siipola, and Annukka Ruokolainen for excellent technical assistance, and FIMM Technology Center for Sanger sequencing services.

Author contributions EAMH and SP conducted the molecular laboratory experiments and analyzed the results (except the telomere analysis). AN and SD conducted the telomere length analysis and analyzed the results. KH-J and HV are responsible for the dermatological and odontological and oral mucosal examination and interpretation, respectively. OK and UW-K designed the study, participated in the analyses, and finalized the manuscript. All authors participated in drafting the manuscript and have read and approved the final manuscript.

\section{Compliance with ethical standards}

Conflict of interest The authors declare that they have no conflict of interest.

\section{References}

1. Savage SA. Beginning at the ends: telomeres and human disease. F1000Res. 2018;7:524 https://doi.org/10.12688/f1000research. 14068.1.

2. Alter BP, Giri N, Savage SA, Rosenberg PS. Cancer in the National Cancer Institute inherited bone marrow failure syndrome cohort after fifteen years of follow-up. Haematologica. 2018;103:30-9.
3. Tukiainen T, Villani AC, Yen A, Rivas MA, Marshall JL, Satija $\mathrm{R}$, et al. Landscape of $\mathrm{X}$ chromosome inactivation across human tissues. Nature. 2017;550:244-8.

4. Devriendt K, Matthijs G, Legius E, Schollen E, Blockmans D, Van Geet $\mathrm{C}$, et al. Skewed X-chromosome inactivation in female carriers of dyskeratosis congenita. Am J Hum Genet. 1997;60:581-7.

5. Vulliamy TJ, Knight SW, Dokal I, Mason PJ. Skewed Xinactivation in carriers of X-linked dyskeratosis congenita. Blood. 1997;90:2213-6.

6. Trotta L, Norberg A, Taskinen M, Beziat V, Degerman S, Wartiovaara-Kautto U, et al. Diagnostics of rare disorders: wholeexome sequencing deciphering locus heterogeneity in telomere biology disorders. Orphanet J Rare Dis. (in press).

7. Hindson BJ, Ness KD, Masquelier DA, Belgrader P, Heredia NJ, Makarewicz AJ, et al. High-throughput droplet digital PCR system for absolute quantitation of DNA copy number. Anal Chem. 2011;83:8604-10.

8. Xu J, Khincha PP, Giri N, Alter BP, Savage SA, Wong JM. Investigation of chromosome $\mathrm{X}$ inactivation and clinical phenotypes in female carriers of DKC1 mutations. Am J Hematol. 2016;91:1215-20.

9. Fernandez-Garcia I, Marcos T, Munoz-Barrutia A, Serrano D, Pio $\mathrm{R}$, Montuenga LM, et al. Multiscale in situ analysis of the role of dyskerin in lung cancer cells. Integr Biol (Camb). 2013;5:402-13.

10. Cawthon RM. Telomere measurement by quantitative PCR. Nucleic Acids Res. 2002;30:e47.

11. Vulliamy TJ, Kirwan MJ, Beswick R, Hossain U, Baqai C, Ratcliffe A, et al. Differences in disease severity but similar telomere lengths in genetic subgroups of patients with telomerase and shelterin mutations. PLoS ONE. 2011;6:e24383.

12. Alder JK, Hanumanthu VS, Strong MA, Dezern AE, Stanley SE, Takemoto CM, et al. Diagnostic utility of telomere length testing in a hospital-based setting. Proc Natl Acad Sci USA. 2018;115: E2358-65.

13. Norberg A, Rosen A, Raaschou-Jensen K, Kjeldsen L, Moilanen JS, Paulsson-Karlsson Y, et al. Novel variants in Nordic patients referred for genetic testing of telomere-related disorders. Eur $\mathbf{J}$ Hum Genet. 2018;26:858-67.

14. Kirschner M, Maurer A, Wlodarski MW, Ventura Ferreira MS, Bouillon AS, Halfmeyer I, et al. Recurrent somatic mutations are rare in patients with cryptic dyskeratosis congenita. Leukemia. 2018. https://doi.org/10.1038/s41375-018-0125-x. 